\title{
Proceeding
}

10th INSHS International Christmas Sport Scientific Conference, 4-5 December 2015. International Network of Sport and Health

Science. Szombathely, Hungary

\section{Physical activity effects on postural adjustments: a review}

\author{
ANDIS BOGDANI ${ }^{1}$, GENTI PANO ${ }^{2}$ \\ ${ }^{1}$ Department of Physical Activity Recreation and Tourism, Sports University of Tirana, Tirana, Albania \\ ${ }^{2}$ Department of Physical Activity Health and Recreation, Sports University of Tirana, Tirana, Albania
}

\begin{abstract}
Postural control has been viewed as an example of the famous problem of motor redundancy (Bernstein, 1967 ) in this case formulated as the problem of coordinated changes in the activation of numerous muscles of the lower extremities and the trunk. Muscular stability and mobility, skeletal structure, and muscular balance all influence postural alignment (Kendall, F.P et al., 1983; Fortin, C., 2011). Depending on the severity of the postural deviations, poor posture may result in pain and can affect physical function and the ability to perform activities of daily living (Katzman, W.B., 2007). Aim of this review was searching studies focusing on the effects physical activity on postural adjustments. We searched PubMed, ResearchGate, JabRef. From 166 studies only 6 fulfilled the inclusion criteria's. A total of 150 subjects with age ranging from 22-61 years old participating in different exercise intervention programs. In general, there are few studies focusing on the effects of physical activity on postural control and adjustments and also all the studies have enrolled a low number of subject which lead to a necessity for further research with larger groups of subjects and different type of physical activity intervention programs in order to better identify which is the most effective. Key words: PHYSICAL ACTIVITY; POSTURE, POSTURAL ADJUSTMENTS.
\end{abstract}

\footnotetext{
Corresponding author. Sports University of Tirana, Sport Science Research Institute, Department of Physical Activity Health and Recreation, Tirana Albania.

E-mail: genti.pano@outlook.com

10th INSHS International Christmas Sport Scientific Conference, 4-5 December 2015. International Network of Sport and Health Science. Szombathely, Hungary.

JOURNAL OF HUMAN SPORT \& EXERCISE ISSN 1988-5202

(c) Faculty of Education. University of Alicante

doi:10.14198/jhse.2016.11.Proc1.15
} 


\section{INTRODUCTION}

Quiet standing balance has been described as the control of a multi-link pendulum (Creath $R$ et al., 2005) that uses the movement of all major body segments to stabilize the center of mass (Hsu WL et al., 2007). Compensatory postural adjustments have also been described as the control of a multi-link pendulum (Horak FB et al., 1986) in which all body segments assist in maintaining stability. Movement is a complex process in which the central processing system integrates and processes information and the musculoskeletal system responds to its (Nashner LM., 1989) static and dynamic location senses, in other words, proprioceptive senses, are known to maintain the stability and orientation of the body during movement (Laskowski ER et al., 2000).

Postural control has been viewed as an example of the famous problem of motor redundancy (Bernstein, 1967) in this case formulated as the problem of coordinated changes in the activation of numerous muscles of the lower extremities and the trunk. Muscular stability and mobility, skeletal structure, and muscular balance all influence postural alignment (Kendall, F.P et al., 1983; Fortin, C., 2011). Depending on the severity of the postural deviations, poor posture may result in pain and can affect physical function and the ability to perform activities of daily living (Katzman, W.B., 2007).

For biomechanics, the standing posture is the result of a dynamic state of balance between body and gravity, which is an external force that attracts the body to the ground. Thus, contraction of the posture muscles and continuous positioning of body segments occur during standing in order to maintain balance and to overcome the action of the gravitational force (Bertoncello D et al., 2009; Benda BJ et al., 1994; Contri DE).

\section{Objectives}

Main objective of this paper was: To review the recent literature regarding physical activity effects on postural adjustments.

\section{MATERIAL AND METHODS}

The search was made mainly focusing in: PubMed, ResearchGate, for studies focusing on the effects physical activity on postural adjustments. Key Words: Physical activity; Posture, Postural adjustments.

Inclusion Criteria for searching were:

- Randomized Controlled Studies.

- Studies in English Language.

- All group ages.

- Studies focusing on the effects physical activity on postural adjustments.

- Studies that have included in their protocol different type of physical activity interventions.

\section{RESULTS AND DISCUSSION}

From 1063 studies only 5 fulfilled the inclusion criteria's. A total of 332 subjects with age ranging from 11-45 years old participating in different exercise intervention programs.

Papers fulfilled the inclusion criterias: 
1. Hwang et al., 2013

2. Vidal et al., 2013

3. Saito et al., 2014

4. Torlakovic et al., 2014

5. Kim et al., 2015

In general, there are few studies focusing on the effects of physical activity on postural control and adjustments and also all the studies have enrolled a low number of subject which lead to a necessity for further research with larger groups of subjects and different type of physical activity intervention programs in order to better identify which is the most effective.

Table 1. Included studies

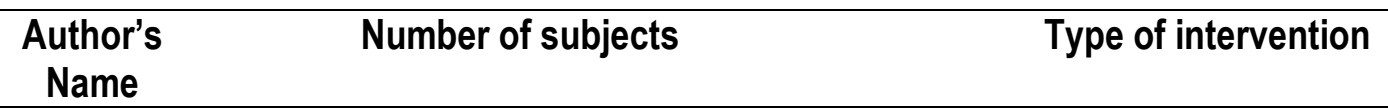

1. Hwang et Total 21 patients. 4 healthy people

al., 2013 and 14 CLBP patients were randomly assigned to Group II (ordinary physical therapy, $n=7$ ) and Group III (sensorimotor training, $n=7$ )

(Group II) to whom ordinary physical therapy was applied and an experimental group (Group III), the members of which received sensorimotor training. For ordinary physical therapy, a hot compress (20 minutes), ultrasound $(1.5 \mathrm{~W} / \mathrm{cm} 2$, five minutes, Jireh Medical, Korea), and transcutaneous electrical nerve stimulation (4 pps, 15 minutes, Hanawoo Medical, Korea) were applied to the L1-2 and L4-5 areas for 40 minutes each time, five times per week, for a total of four weeks. A total of six kinds of exercises were conducted for 40 minutes each time, five times per week, for four weeks.

2. Vidal et al., 137 children aged 10.7 years $(S D=$ 2013 0.672). Six classes from two primary schools were randomly allocated into experimental group $(E G)(N=63)$ or control group (CG) $(\mathrm{N}=74)$.

The EG received a postural education program over 6 weeks consisting of six sessions, while the CG followed the usual school curriculum. A questionnaire was fulfilled by the participants at pre-test, post-test, and 3 months after the intervention finished.

3. Saito et al., $16(22.6 \pm 2.1$ years; height $(65.7 \pm$ 2014

9.5; weight: $58.8 \pm 7.0 \mathrm{~kg}$

4. Torlakovic 70 girls, aged $11.9 \pm 2.3$ years et al., 2014

Subjects stood barefoot on a force platform and reached as quickly and accurately as possible to a target placed at their maximum reach distance immediately following a beep signal in a reaction time condition. Whole-body reaching training with the right arm was repeated 100 times for three consecutive days.

The subjects were included in the program of kinesiology treatment with duration of 28 weeks. 
5. Kim et al., 88 students from $S$ University in K city 2015 (male students, $\mathrm{n}=34$; female students, $n=54$ ).
The exercise program for posture correction was performed for 20 minutes per session, 3 times a week for 8 weeks. Pain levels were measured using a pain scale, and pain levels before and after the exercise program were compared.

1. Hwang et al., 2013: "The Effects of Sensorimotor Training on Anticipatory Postural Adjustment of the Trunk in Chronic Low Back Pain Patients" A total of 21 patients were enrolled in this study. 7 healthy people and 14 CLBP patients were randomly assigned to Group II (ordinary physical therapy, $n=7$ ) and Group III (sensorimotor training, $\mathrm{n}=7$ ). (Group II) to whom ordinary physical therapy was applied and an experimental group (Group III), the members of which received sensorimotor training. For ordinary physical therapy, a hot compress (20 minutes), ultrasound $(1.5 \mathrm{~W} / \mathrm{cm} 2$, five minutes), and transcutaneous electrical nerve stimulation ( 4 pps, 15 minutes) were applied to the L1-2 and L4-5 areas for 40 minutes each time, five times per week, for a total of 4 weeks. A total of six kinds of exercises were conducted for 40 minutes each time, five times per week, for four weeks.

2. Vidal et al., 2013: "Effects of a postural education program on school backpack habits related to low back pain in children". A total of 137 children aged 10.7 years $(S D=0.672)$. Six classes from two primary schools were randomly allocated into experimental group $(E G)(N=63)$ or control group $(C G)(N=74)$. The EG received a postural education program over 6 weeks consisting of six sessions, while the CG followed the usual school curriculum. A questionnaire was fulfilled by the participants at pre-test, post-test, and 3 months after the intervention finished.

3. Saito et al., 2014: "Relationship between improvements in motor performance and changes in anticipatory postural adjustments during whole-body reaching training". This study aimed to investigate the relationship between improvement in motor performance and changes in APAs during repeated reaching training, as well as the learning effects on APA changes. A total of 16 subjects ( $22.6 \pm 2.1$ years; height $(65.7 \pm 9.5$; weight: $58.8 \pm 7.0 \mathrm{~kg}$. Subjects stood barefoot on a force platform and reached as quickly and accurately as possible to a target placed at their maximum reach distance immediately following a beep signal in a reaction time condition. Whole-body reaching training with the right arm was repeated 100 times for three consecutive days. Motor performance and APAs were evaluated on the first day, after discontinuation of training for one day, and again at three months.

4. Torkalovic et al., 2014: "Evolution of Sports-medical Team Management in the Program of Posture Correction in Children". A total of 70 girls, aged 11.9-2.3 years, in which by the expert evaluation is recorded weakness of individual muscle groups, but also of the whole musculature. For the assessment of posture, they applied the method of Napoleon Wolanski. This method determines the deviations in keeping body parts from normal (regular) posture. The subjects were included in the program of kinesiology treatment with duration of 28 weeks.

5. Kim et al., 2015: "Effect of an exercise program for posture correction on musculoskeletal pain". The present study investigated the effect of an exercise program for posture correction on musculoskeletal pain. An exercise program was performed in 88 students from $S$ University in $\mathrm{K}$ city (male students, $n=34$; female students, $n=54$ ). The exercise program for posture correction was performed for 20 minutes per session, 3 times a week for 8 weeks. Pain levels were measured using a pain scale, and pain levels before and after the exercise program were compared. 


\section{CONCLUSIONS}

Based on the reviewed studies we can conclude that:

In general, there are few studies focusing on the effects of physical activity on postural control and adjustments. All studies have enrolled a low number of subject which lead to a necessity for further research with larger groups of subjects and different type of physical activity intervention programs in order to better identify which is the most effective.

According to Hwang et al., 2013, Sensorimotor training makes patients capable of learning how to adjust muscles, thereby alleviating pain and improving muscle performance.

According to Vidal et al., 2013, study findings confirm that children are able to learn healthy backpack habits which might prevent future low back pain.

In Saito et al., 2014. study have found that changes in Anticipatory Postural Adjustments induced by repetitive reaching training occurred earlier than improvements in reaching performance, and that the training effects were retained after subjects discontinued training.

These findings suggest that the Central Nervous System may be able to adapt Anticipator Postural Adjustments for improvement in motor performance. Therefore, a better understanding of postural control mechanisms in patients with postural instability may be useful for physical therapy practices.

Future research should address the contribution of the Central Nervous System to changes in postural control during repetitive movement.

In Torkalovic et al., 2014 study, results showed that the models which complement the experience and practical application of expert health professionals and kinesiology knowledge is a very effective tool for improving posture of girls in the second phase of intensive growth and development.

According to Kim et al., 2015 results and conclusions, the overall, pain levels of the participants were lower after the exercise program than before the program, and significant differences in pain levels were noted in the shoulders, middle back, and lower back-shoulder pain, mid back pain, and low back pain were relieved with the exercise program for posture correction. Therefore, the findings of this study can be used to improve the work efficiency of students as well as people engaged in sedentary work.

\section{REFERENCES}

1. Benda, B.J., Riley, P.O., \& Krebs, D.E. (1994). Biomechanical relationship between center of gravity and center of pressure during standing. IEEE Trans. Rehabil. Eng., 2, 3-10.

2. Bernstein, N.A. The coordination and regulation of movements. Pergamon Press; London: 1967.

3. Bertoncello, D., Sá, C.S., Calapodópulos, A.H., \& Lemos, V.L. (2009). Equilibrium and muscle retraction in young female student's users of high-heeled shoes. Fisioter. Pesq., 16, 107-12.

4. Contri, D.E., Petrucelli, A., \& Perea, D.C. (2009). Postural deviation incidence in students of the 2 nd to 5 th year of the elementary school. ConScientiae Saude, 8, 219-24.

5. Fortin, C., et al. (2011). Clinical methods for quantifying body segment posture: A literature review. Disabil. Rehabil., 33(5), 367-83. 
6. Katzman, W.B. (2007). Changes in flexed posture, musculoskeletal impairments, and physical performance after group exercise in community—dwelling older women. Arch. Phys. Med. Rehabil., 88(2), 192-9.

7. Kendall, F.P., McCreary, E.K. Muscles, Testing and Function, 3rd ed. 1983, Baltimore: Lippincott, Willams \& Wilkins.

8. Laskowski, E.R., Newcommer-Aney, K., Smith, J., et al. (2000). Proprioception. Phys. Med. Rehabil. Clin. N. Am., 11, 323-340.

9. Nashner, L.M. (1989). Sensory, neuromuscular, and biomechanical contributions to human balance. In: Duncan PW, ed. Balance, Proceedings of the APTA Forum, American Physical Therapy Association. Alexandria, Virginia, pp 5-12. 